Página inicial: 397 - Página final: 417

TIPO DE ARTÍCULO: de Revisión

\title{
PLAN PROSPECTIVO PARA EL DESARROLLO AGRARIO EN LAS REGIONES COLOMBIANAS A PARTIR DEL POSCONFLICTO AL AÑO 2025.
} PROSPECTIVE PLAN FOR THE AGRICULTURAL DEVELOPMENT IN THE COLOMBIAN
REGIONS STARTING FROM THE POST-CONFLICT UP TO THE YEAR 2025.

\author{
Recibido: Enero 2014 Revisado: Marzo2014 Aceptado: 1 de Mayo 2014
}

\author{
Por: Efrain Guerrero Sanchez ${ }^{1}$, Angel Andres Rojas Peña ${ }^{2}$, Maria Yolanda \\ Torres $^{3}$, Nubia Alexandra Bourdon Rojas ${ }^{4}$.
}

\section{Resumen.}

El presente texto derivado de investigación, presenta los resultados del análisis prospectivo para el desarrollo agrario de las regiones colombianas, de cara a un posible acuerdo fruto de las actuales (2014) negociaciones de Paz entre la guerrilla de las Farc-EP y el Gobierno Colombiano, acuerdo que de firmarse permitiria a la sociedad Colombiana entrar en lo que por ahora se ha denominado posconflicto. En este texto se proyectan posibles escenarios al año 2025; nos planteamos 4 posibles escenarios, dos alternos identificados como: Bienestar a Media y Sobreviviendo, un escenario catastrófico denominado Pobreza Absoluta y nuestro escenario apuesta: Paz y Prosperidad.

\section{Palabras claves.}

Prospectiva, Escenarios, Posconflicto, Paz, Territorios, Desarrollo.

\section{Abstract.}

The current text, which was derived from research, introduces t he results of the prospective analysis for the agricultural development in the Colombian regions, with a view of a possible agreement, as a result of the current (2014) peace negotiations between the FARC-EP guerrilla forces and the Colombian government, an agreement which, in the event of its being signed, would let the Colombian society enter into what by now has been called post-conflict. In this text, some possible scenarios are projected to the year 2025; four possible scenarios, two alternate ones, which are identified as: Average Welfare and Surviving, a catastrophic scenario known as Absolute Poverty, and our bet stage: Peace and Prosperity.

\section{Key words.}

Prospective; Stages; Post-Conflict; Peace; Territories, and Development.

\footnotetext{
${ }^{1}$ Administrador de Empresas, investigador Universidad Nacional Abierta y a Distancia UNAD. Adelanta estudios Doctorales en el IPECAL de México. Actualmente se desempeña como Gestor de Paz. Medellín, Colombia. Contacto: efrain.guerrero@unad.edu.co, efrainguerrerosanchez@hotmail.com .

${ }^{2}$ Docente investigador Universidad Nacional Abierta y a Distancia UNAD. Medellín-Colombia. Contacto: Andres.rojas@unad.edu.co.

${ }_{3}^{3}$ Docente investigadora Universidad Nacional Abierta y a Distancia UNAD, Medellín-Colombia. Contacto: Maria.torres@unad.edu.co .

${ }^{4}$ Docente investigadora Universidad Nacional Abierta y a Distancia UNAD, Medellín-Colombia. Contacto .Nubia.bourdon@unad.edu.co.
} 


\section{Introducción.}

El sector agrario, un sector importante para la economía, se encuentra en crisis, lo que es curioso en un país con un inmenso potencial agrícola como Colombia.

Abordar el tema del desarrollo agrario en una posible etapa de posconflicto (Mesa de Conversaciones Habana., 2013) es una necesidad, por tal razón buscamos por medio de esta investigación, diseñar un plan prospectivo y estratégico que permita el desarrollo agrario de las regiones colombianas a partir del postconflicto.

Colombia posee un potencial agrícola que no ha sido aprovechado. Según el último informe nacional de desarrollo humano 2011, elaborado por el PNUD nos muestra esta realidad:

Para el año 2009 se identifica un fenómeno de gran subutilización de la tierra apta para el desarrollo de cultivos. La superficie dedicada a actividades agrícolas y silvoagrícolas asciende a 4,9 millones de hectáreas, pese a que se estima que 21,5 millones tienen aptitud agricola; es decir, solo el 22,7\% de la superficie con esa vocación es utilizada para cultivos (Pnud, 2011).

Colombia hace parte de la llamada "despensa agrícola" latinoamericana, que puede ofrecer soluciones reales al incremento de la demanda por alimentos que se vive en la actualidad. A pesar de esto el sector agrícola en Colombia está en crisis, trayendo como consecuencia la quiebra de los pequeños y medianos agricultores aumentando las cifras de pobres en el país, que según cifras del Banco Mundial, la población rural que vive por debajo de la linea de pobreza rural nacional en Colombia es del 46,8 \%. (Banco Mundial, 2013)

La importancia que tiene para la economía nacional el desarrollo del sector agrario no es menor, estamos hablando del sector de la economía que está ocupando en la actualidad según el DANE, el tercer lugar, con un aporte al PIB de 11,3 $\%$. Representada en una población que según el informe nacional de desarrollo humano 2011 del PNUD, "no el 25 sino el 32\% de los colombianos son pobladores rurales" (Pnud, 2011). Así las cosas el desarrollo agrario de las regiones colombianas adquiere mayor relevancia en estos momentos ante la gran expectativa que despierta los resultados de los diálogos de paz gobierno y grupos insurgentes, posiblemente su incidencia cambiara la historia del país.

\section{El conflicto insurgente y el sector agrario en Colombia.}

El conflicto interno colombiano ha sido la excusa para el abandono del campo, por lo que se dice que el desarrollo del sector agrario está ligado a la superación de este conflicto y razón se tiene si miramos que el primer punto en la agenda de negociaciones gobierno FARC EP así lo demuestra.

....hemos llegado a un acuerdo sobre el primer punto de la Agenda contenida en el Acuerdo General para la Terminación del Conflicto y la Construcción de una paz Estable y Duradera, que acordamos denominar "Hacia un nuevo campo colombiano: Reforma Rural Integral". (Mesa de Conversaciones Habana., 2013) 
Así las cosas en lo que se ha llamado reforma rural integral estaría planteada la politica para el desarrollo agrario en Colombia. En tal sentido se proponen instrumentos como la implementación de las llamadas zonas de reserva campesina, la creación de un fondo de tierras, la puesta en marcha por parte del gobierno de un plan para formar y actualizar el catastro rural, la creación de una figura llamada Jurisdicción agraria hecha entre otras cosas para la conciliación y resolución de conflictos.

Como vemos, el futuro del sector agrario colombiano y puntualmente en de los pobladores rurales, depende de lo acordado en las mesas de diálogo con las insurgencias, bien sabemos que las distintas expresiones organizativas representantes de los pobladores rurales enviaron sus puntos de vista a la mesa de negociaciones de la Habana, ejercicio que hicieron a través de foros de participación realizados por la Universidad Nacional y un grupo de integrantes del congreso de la república.

\section{El Sector Agrario En El Posconflicto Salvadoreño Y Guatemalteco.}

Caso salvadoreño. Cuando se logran los acuerdos de paz en el año 1.992 en Chapultepec México, uno de los principales temas fue la reactivación agrícola. Como resultado de los acuerdo se creó el Banco de Tierras, ente estatal que se encargaría de legalizar la tenencia de la tierra, para que los campesinos y los excombatientes del conflicto pudieran trabajarla, se prometió condonación parcial de la deuda agraria y bancaria entre otras politicas de reactivación.

Lo que parecía un futuro prometedor fue un rotundo fracaso, el congreso, en el período anterior 1,997-2,000, por decreto legislativo decidió cerrar el Banco de Tierras, y las condonaciones de las tierras tampoco se cumplieron. Terminado el conflicto el sector agrario salvadoreño no repuntaba, miremos unas cifras al respecto para mayor claridad.

La investigación de Acevedo, Padilla y Ramos (1999) pg. 101-107), plantea que de acuerdo a cifras oficiales, el sector agropecuario creció 0.2 por ciento en 1998, manteniendo la tendencia decreciente mostrada desde 1995 (4.5\% en $1995,1.2 \%$ en 1996 y $0.9 \%$ en 1997). Una clara manifestación de los cambios experimentados por la economía salvadoreña desde los años ochenta, la constituye la pérdida de importancia relativa del sector agropecuario dentro del PIB. Durante el período 1990-97, la contribución porcentual de las actividades agropecuarias al PIB real fue de $15 \%$, comparada con un promedio de $24.4 \%$ durante 1970-79. De acuerdo a FUSADES (2001, págs. 33-35), la evolución del sector agropecuario a lo largo de la década de los noventa se caracterizo por altibajos en su tasa de crecimiento anual, la cual fue incluso negativa en 1993, 1994, 1998 y 2000. Los rubros más dinámicos fueron la caña de azúcar (6.9\%), otras producciones agrícolas (3.7\%) y granos básicos (1.6\%). El café muestra una tasa negativa de crecimiento promedio anual (-1.4\%). Entre los pecuarios, el rubro más dinámico fue la avicultura (5.2\%), seguido de la ganadería $(1.7 \%)$. (Adepro, 2001)

Lo que se ha podido ver es que el sector agrícola en El Salvador es uno de los sectores menos protegidos a tal grado que después de ser uno de los principales proveedores de materia prima a otros países, en la actualidad se tiene que importar muchos productos 
provenientes de la tierra ya que cada vez es menos el número de personas dedicadas a las actividades agrícolas. Las medidas tomadas por el gobierno después del posconflicto como la privatización de la banca, la dolarización de la economía central del país, lineas de financiamiento para inversiones nacionales, y otros proyectos de inversión, únicamente han beneficiado al sector comercial y al de la banca comercial, ninguno de ellos ha beneficiado de forma directa al sector agrícola.

Caso Guatemala. Guatemala vivió 36 años de conflicto, firmando acuerdos de paz en el año 1996. Es así como en lo relacionado con el tema de acuerdo sobre aspectos socioeconómicos y situación agraria, encontramos que:

....Éstos incluían acceso a la propiedad de la tierra, construcción de infraestructura básica, modernización de las modalidades de producción y del cultivo, acceso a crédito y servicios financieros, capacitación y asistencia técnica, acceso a información, protección laboral, uso sostenible de los recursos del territorio. (Alasdair , 2005)

Una evaluación sobre el avance de los acuerdos del proceso de paz en Guatemala realizada por una Delegación guatemalteca para la incidencia internacional conformada por: Gilberto A, Byron G, Úrsula R, nos dice que "el Estado no ha cumplido con el rol asignado por los Acuerdos de Paz" (Adepro, 2001). En el posconflicto se ejecutó una política de disminución del gasto público, de privatización y consecuentemente se desmantelaron casi la totalidad de instituciones de servicio para el sector agrícola.

Esta misma delegación encontró que "La política que está siendo aplicada da prioridad a los sectores "productivos" entendiéndose por ellos a los grandes terratenientes y sector agroexportador, dejando a su suerte a los pequeños productores a quien sólo se les asigna los fondos sociales. (Adepro, 2001)

En lo que tiene que ver con la tan nombrada reforma agraria acordada para el posconflicto, tampoco funciono, por el contrario la acumulación de la tierra en manos de terratenientes creció. "Menos del $1 \%$ de los productores posee casi el $75 \%$ del área total de tierra cultivable" (Alasdair, 2005). Los pequeños agricultores llevan una vida precaria; no tiene infraestructura básica, acceso a crédito o acceso a los mercados.

"se ha aumentado la pobreza rural; muchos agricultores han abandonado la tierra y se han mudado a las áreas urbanas ya sobre pobladas; otros, en búsqueda de fuentes alternativas de ingresos, están talando bosques por madera" (Alasdair , 2005)

Al interpretar a Cunningham, A. (2005) "Durante los años después de los Acuerdos de paz, el Ministerio de Agricultura fue uno de los muchos sectores públicos que se desmantelaron, se redujo de 22.000 a sólo 800 empleados". Una clara demostración de la falta de voluntad para el cumplimiento de los acuerdos pactados.

Guatemala a pesar de ser un país con un gran potencial agrícola, hoy está importando algunos alimentos, que podrían ser producidos en sus suelos pero estos están ocupados con grandes proyectos de monocultivos. 


\section{Tendencias Mundiales.}

Las tendencias mundiales con respecto al desarrollo del sector agrario en los posconflictos nos muestra una tendencia poco optimista para el desarrollo integral del campo. Los modelos de desarrollo para el sector agrario no están pensados para resolver los problemas de los pobladores ruarles, aunque se diseñan así esto no se cumple, y se termina es abriéndole el espacio a las multinacionales explotadoras de recursos naturales y las grandes empresas nacionales productoras de alimentos y biocombustibles.

De otro lado vemos como en la gran mayoría de los países africanos donde se desarrollan históricos conflictos, la tendencia ha sido el de resolver los conflictos para apoderarse de los recursos naturales existentes en sus territorios y poco les importa la catástrofe humanitaria que dejan los conflictos y la devastación de sus territorios producto de la explotación de sus recursos naturales.

\section{Metodologia.}

Hemos recogido la información para el presente trabajo a través de la elaboración de un estado del arte sobre la situación actual de sector agrario en Colombia, y una mirada al desarrollo agrario en países donde se superaron conflictos similares al nuestro como es caso de Nicaragua y salvador.

Se han consultado un grupo de expertos en el tema agrario, mediante el método Delphi, que han permitido construir a la par de cierta información recogida sobre el tema de desarrollos agrarios en otros países en posconflictos casos concretos Guatemala y El salvador, un plan prospectivo que le busque la materialización de un escenario apuesta llamado paz y prosperidad.

De otro lado hemos utilizado el método Delphi metodología de investigación multidisciplinar para la realización de pronósticos y predicciones. Su funcionamiento se basa en la elaboración de un cuestionario que ha de ser contestado por los expertos, una vez recibida la información, se vuelve a realizar otro cuestionario basado en el anterior para ser contestado de nuevo y finalmente el responsable del estudio elaborará sus conclusiones a partir de la explotación estadística de los datos obtenidos, cuyo objetivo es la consecución de un consenso basado en la discusión entre expertos. Este se realizó mediante la selección de un grupo de expertos en el tema agrario, entre los que contamos dirigentes campesinos y académicos, a los cuales se les pregunto su opinión sobre el tema del sector agrario en Colombia relacionado con el posconflicto.

Como software de apoyo para el análisis de la información recogida, se hizo a través del software MIC MAC - método de análisis estructural de variables que se aplica con la finalidad de establecer las relaciones entre variables, buscando identificar el grado de influencia o dependencia de cada una sobre el resto y MACTOR - método de análisis de juego de actores, que busca valorar las relaciones de fuerza entre los actores y estudiar sus convergencias y divergencias con respecto a un cierto número de posturas y de objetos asociados. A partir de este análisis, el objetivo de la utilización del método MACTOR es el de facilitar a un actor una ayuda para la decisión de la puesta en marcha de su política de alianzas y de conflictos. (Godet, M, 1993) 
Para la construcción de escenarios hemos utilizado como referencia lo planteado por Francisco José Mojica de la Universidad Externado de Colombia, adaptado por Marilú Avendaño de la Universidad Nacional Abierta y a Distancia UNAD, haciendo uso del eje de Peter Schwartz para la construcción de los escenarios futuros, clasificando las variables estratégicas en dos categorías y ubicando cada grupo de variables sobre un eje especifico. Socio politicas (Y) y económico internacionales (X). De tal manera que en el escenario 1 las variables socio políticas influyen positivamente y las variables económico internacionales influyen negativamente. (Schwartz, 1995)

En el escenario 2, Las variables socio politicas influyen negativamente, y las variables económico internacionales influyen positivamente. En el 3 las variables socio políticas influyen positivamente, y las variables económico internacionales influyen también positivamente. (Es nuestro escenario apuesta) Y finalmente en el escenario 4 las variables socio politicas influyen negativamente, las variables económico internacionales influyen también negativamente.

De la misma manera hemos construido una matriz DOFA como herramienta para identificar las debilidades internas, las oportunidades externas, fortalezas internas y amenazas externas de nuestro escenario apuesta. En el análisis de dicha matriz se han construido las estrategias, enfocando los factores claves a tener en cuenta para el desarrollo exitoso de nuestro escenario apuesta.

\section{Resultados - discusiones.}

\section{Método DELPHI.}

Grafica 1: Grado de Importancia de Actores.

\section{Grado de Importancia de Actores}

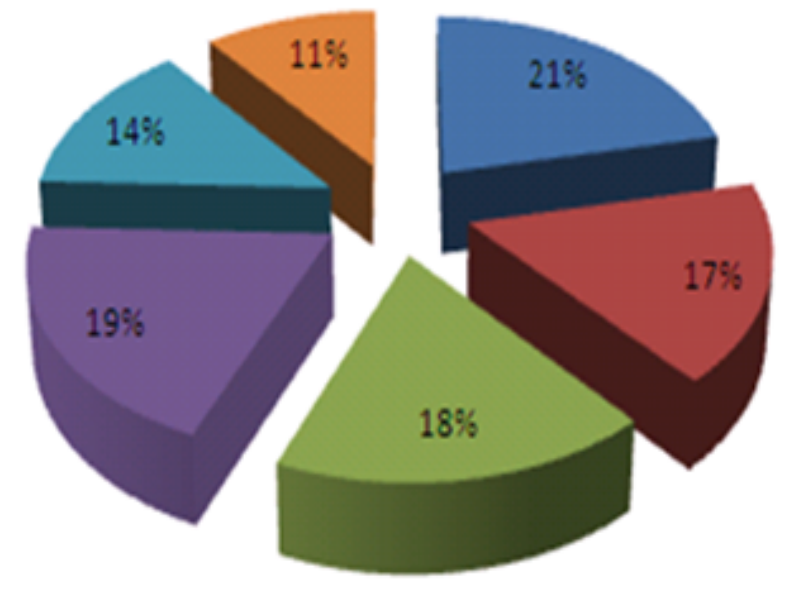

- Campesinos

Insurgentes excombatientes

del conflicto

inremios del sector agrario

Instituciones del estado

Inversionistas del sector

privado

Terratenientes

Fuente: Elaboración propia 
Como resultados de la aplicación del método Delphi encontramos entre otras cosas lo siguiente: se considera como las variables más importantes a tener en cuenta; la inversión en el Sector, el tema de la conservación del medio ambiente y la explotación de recursos naturales.

En cuanto a la importancia de los actores, grado de poder de estos y nivel de compromiso con las politicas para el desarrollo del sector, vemos que son los campesinos los que ocupan el primer lugar, situación que permite determinar que cualquier plan de desarrollo para el campo debe ser elaborado con la participación de los actores más importantes del sector, que son los campesinos.

Consultados sobre los tres principales problemas para la aplicación de nuestro plan prospectivo se considera que son: el modelo actualmente aplicado al campo de saqueo y explotación desmedida de los recursos y los bienes naturales, la concentración de la tierra, y la falta de inversión y atención al sector agrario, tanto por parte del gobierno como por parte del sector privado.

Grafica 2: cómo están acordados hoy los TLCs y otros tratados de integración.

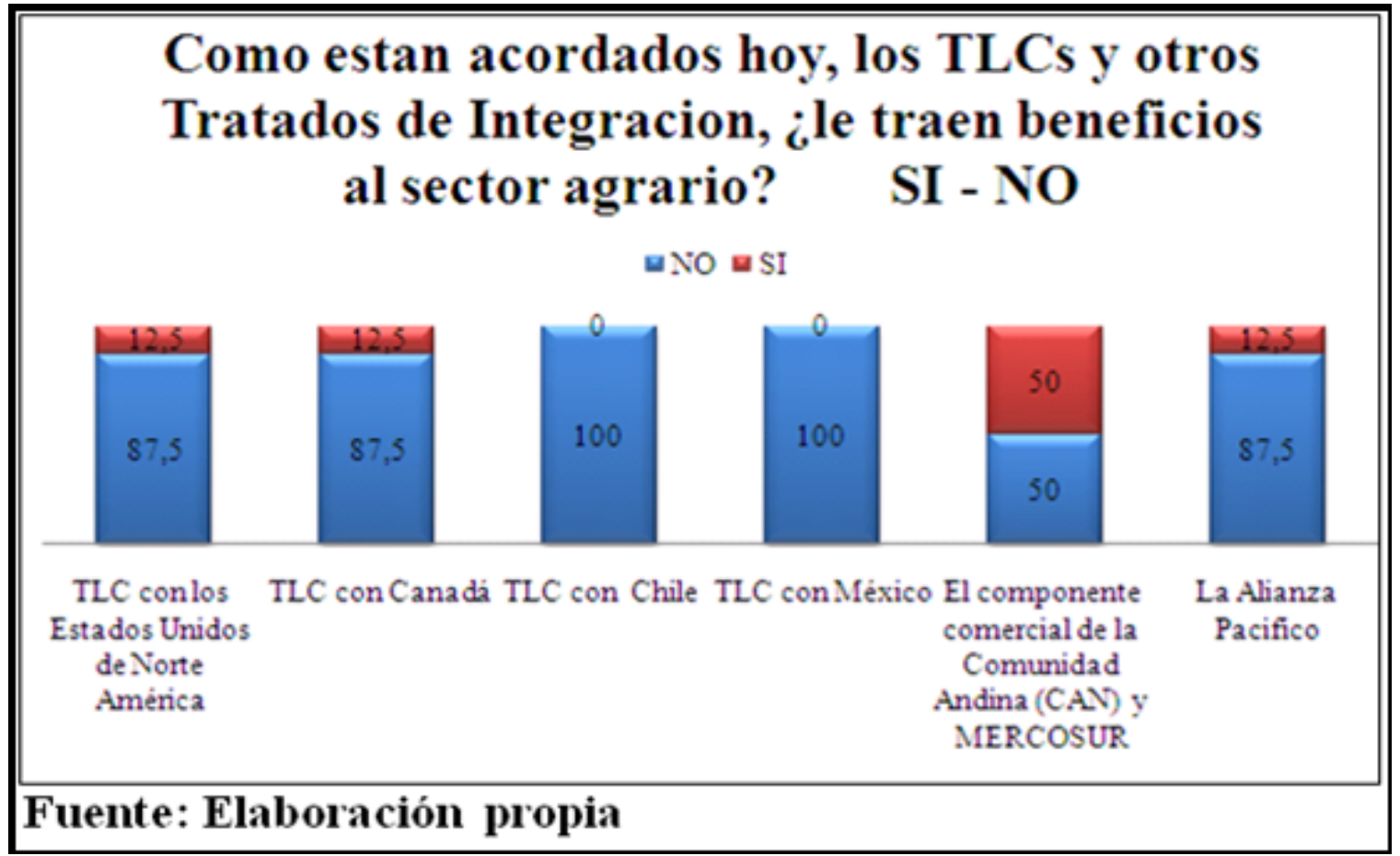

El otro elemento importante tiene que ver con el rechazo a los TLCs. Se considera que tal como están acordados hoy estos tratados no le traen benéficos al sector agrario colombiano.

De otro lado se considera de gran importancia para el desarrollo del campo la finalización del conflicto interno, por lo que se considera supremamente pertinente los diálogos de paz que actualmente se desarrollan en la Habana Cuba. (Garay, L. J., Berberi, F., \& Cardona, I; 2010) 
Método MIC MAC.

Los resultados obtenidos a través del método MIC MAC nos permitió identificar las variables clave o estratégicas las cuales vemos ubicadas en la parte posterior derecha del plano de influencias dependencias / directas, tales como: las políticas estatales para el desarrollo del sector, destinación de recursos para la inversión en el sector, el apoyo

Grafica 3: Plano de influencia / dependencias directas

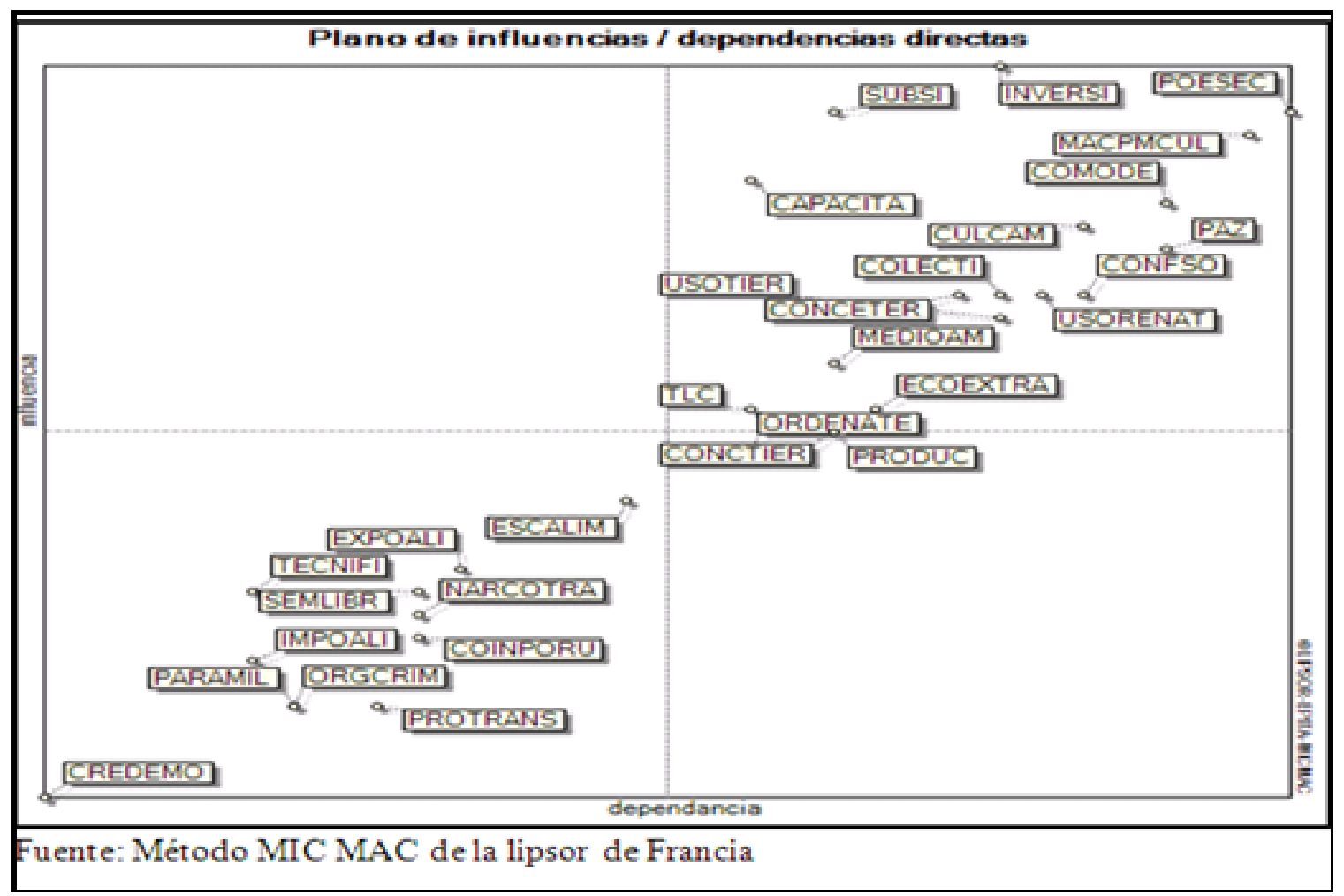

económico a través de subsidios para los productores, la capacitación entendida como la formación intelectual y técnica para los actores, los conceptos de modelos de desarrollo, los macro proyectos de monocultivos, el proceso de paz que sería la solución al conflicto, la cultura campesina, el uso de los recursos naturales, el conflicto social existente entre la sociedad, gobierno y empresas multinacionales, el concepto de territorio, la colectivización de los territorios o la propiedad colectiva de los territorios, el medio ambiente ligado a la conservación de los ecosistemas, el uso de la tierra y de los territorios, la economía extractiva relacionado esto con la explotación indiscriminada de los recursos naturales y la concesión de tierras que tiene que ver con el tema de la restitución de tierras. Estas son las variables más importantes y las llamadas a potencializar por parte de los actores para la materialización de nuestro escenario apuesta.

De otro lado encontramos una importante influencia entre ciertas variables como es el caso de la inversión, con los macroproyectos de monocultivos y las políticas estatales para el desarrollo del sector. Y a su vez las politicas estatales para el 
desarrollo del sector ejercen una gran influencia sobre los subsidios y los proyectos de monocultivos. De ahí la deducción de que las políticas estatales para el desarrollo del sector es la variable más importante según nuestros resultados a través del software MIC MAC, en el entendido de que esta variable dependen la gran mayoría del resto de las variables que hemos considerado claves y estratégicas.

Método MACTOR - juego de actores.

En el plano de influencias y dependencia directas, podemos visualizar claramente en el cuadrante superior derecho, a los actores enlaces tales como comisión de paz, campesinos, Negritudes, Ministerio de Agricultura, Banco Agrario, Sociedad de Agricultores, Excombatientes, Indigenas, Gremios del Sector Agrario y el INCODER. (INCODER, 2013)

Grafica 4: Plano de influencias y dependencias entre actores.

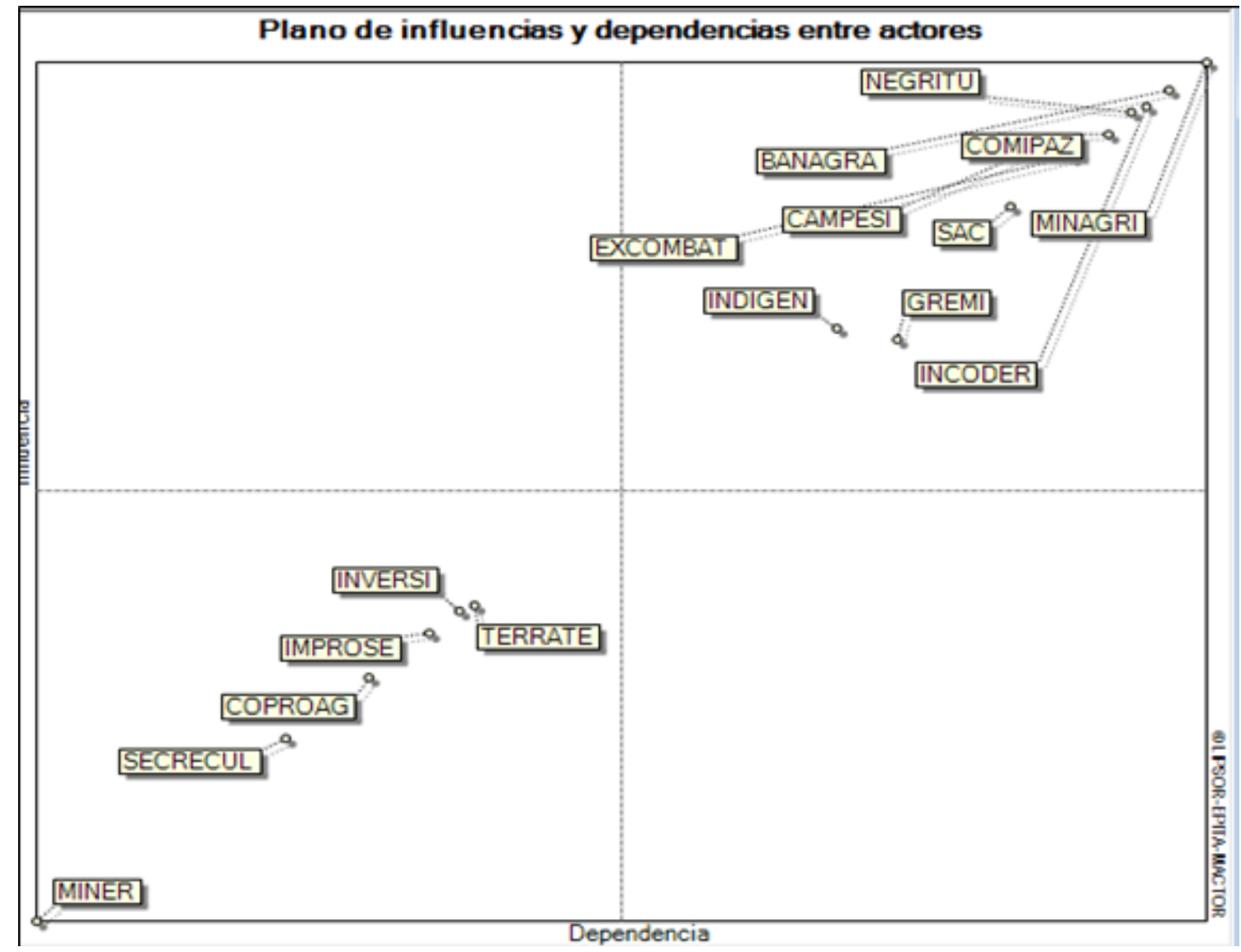

Son llamados actores enlace porque son los actores llamados a manejar las variables claves o estratégicas y son los actores de mayor importancia en el desarrollo del plan prospectivo y que permitirán la consecución del escenario apuesta. 
Gráfica 5: distancias netas entre actores.

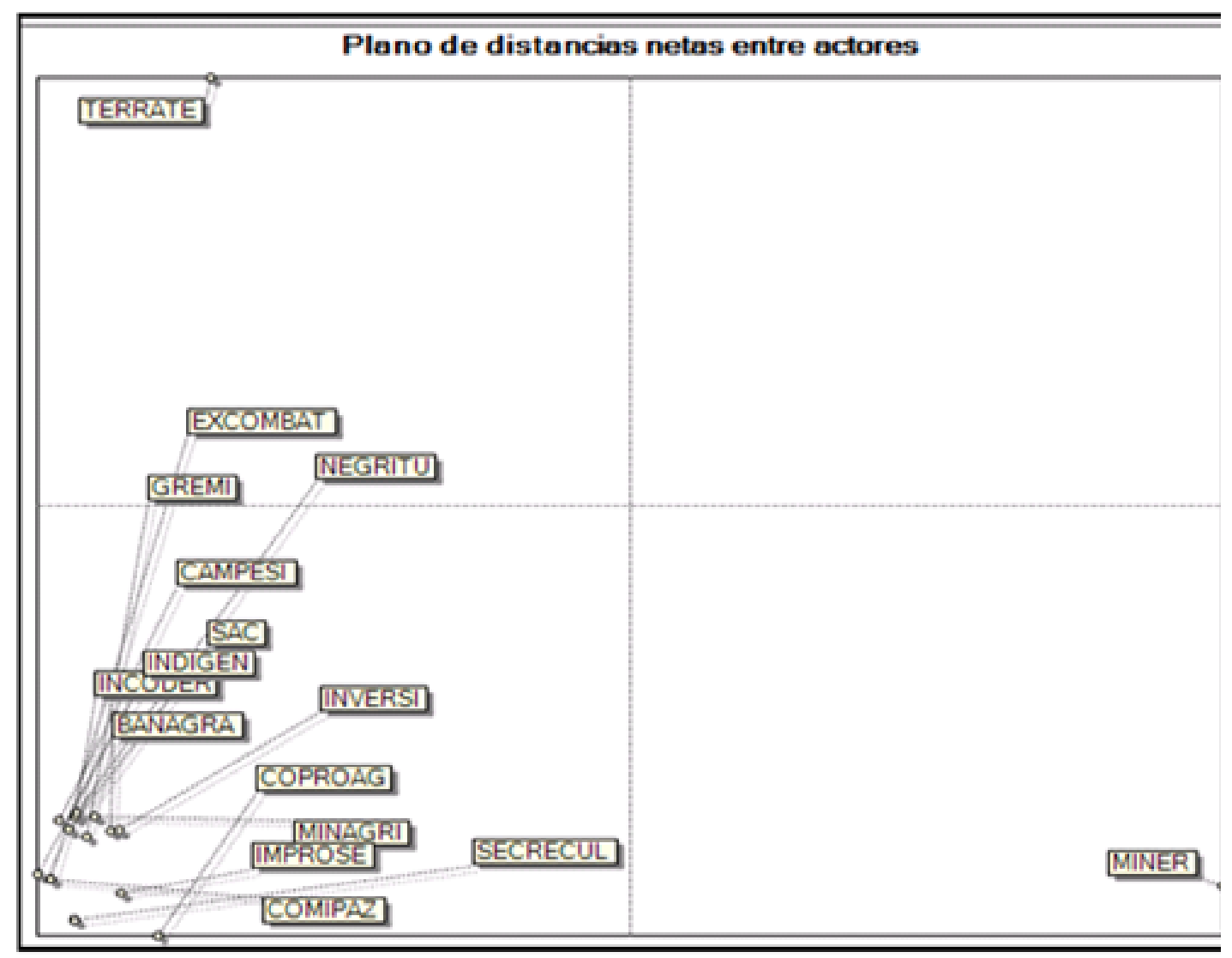

Método MACTOR de la lipsor de Francia

Otro importante resultados lo vemos en el plano de distancias netas entre actores, donde identificamos los actores propensos a generar conflictos, en los que tenemos a los mineros y a los terratenientes. La razón está relacionada con el objetivo de estos actores, el de los terratenientes cuyo objetivo es la acumulación de tierras y que ha sido desaprobado por un gran número de actores. Caso similar con el objetivo de los mineros de extraer los recursos naturales del subsuelo que producen rechazo en ciertos actores con los cuales serian alta probabilidad de generar conflictos.

Pero contario a esto encontramos el caso del objetivo más aceptado por los actores y es el objetivo de la comisión de paz el cual es: promover y garantizar la paz y la reconciliación.

Escenarios.

Nuestro plan prospectivo para el desarrollo agrario de las regiones colombianas a partir del posconflicto, presenta cuatro escenarios; dos escenarios alternativos llamados: Sobreviviendoy Bienestar a Medias, un escenario catastrófico denominado Pobreza Absoluta y nuestro gran escenario apuesta de paz y prosperidad. 
Gráfica 6: escenarios posibles.

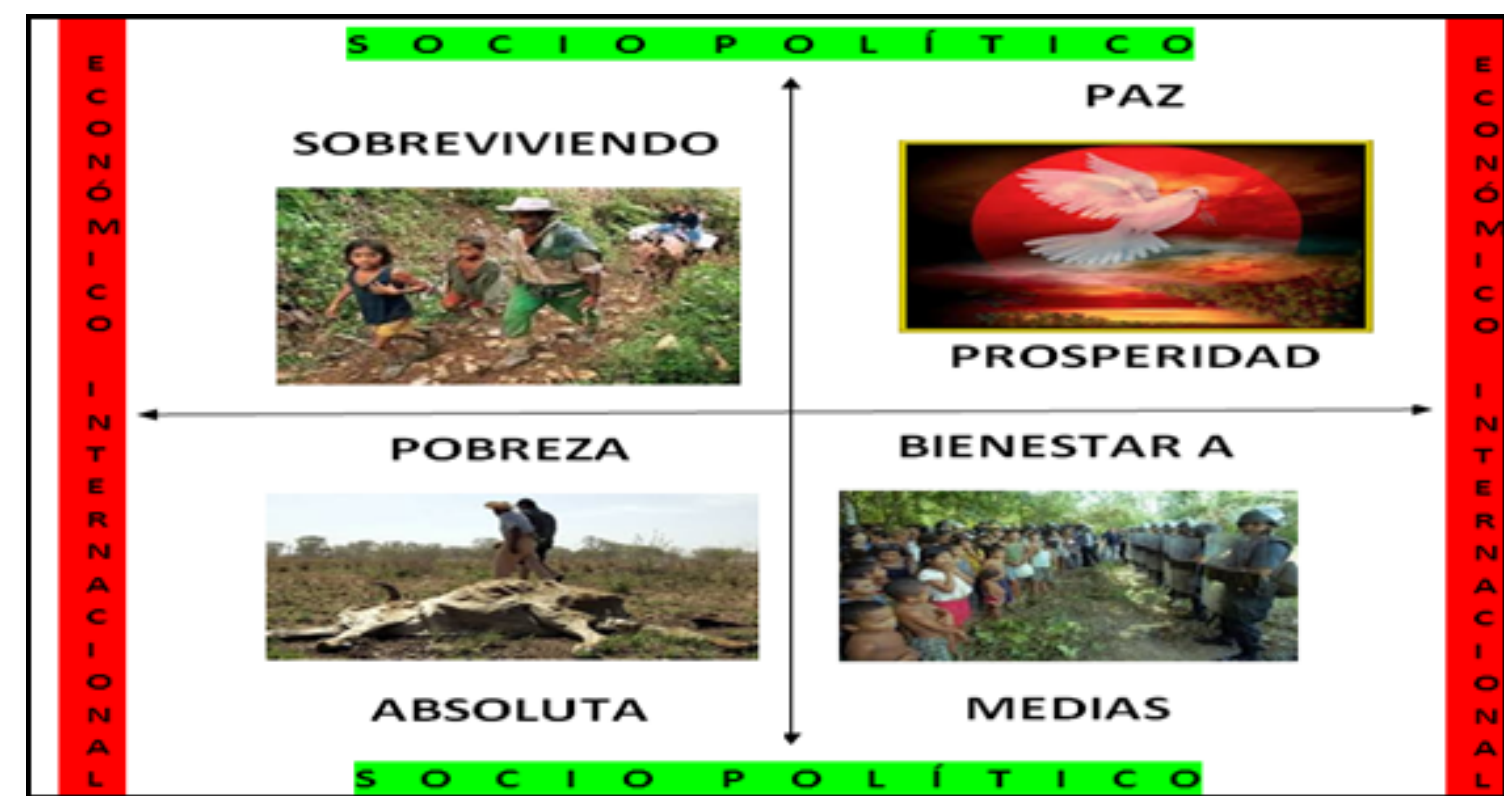

Fuente: Elaboración propia.

Escenario alternativo bienestar a medias.

En este escenario, el campo de lo económico internacional seria exitoso; habría subsidios para los campesinos, inversión del sector privado y de las instituciones del estado como el ministerio de agricultura, el INCODER y demás, e implementación de Macro proyectos de monocultivos, firma y puesta en marcha de TLCS con distintos países razón está de aumento de las exportaciones. (INCODER, 2013)

Las 21,5 millones de hectáreas de tierras aptas para la producción estarian siendo utilizadas. Abría una fuerte explotación de recursos naturales, pues todo esto favorecería el crecimiento económico. (+), Pero en las regiones agrarias colombianas continuarian las dificultades con la definición de políticas estatales serias para el desarrollo Integral de los campos colombianos. Este modelo de desarrollo y crecimiento económico puesto en marcha de explotación masiva de recursos naturales minero energéticos y los macroproyectos para producción de biocombustibles que esterilizan los suelos no serian aceptados por los pobladores rurales, ellos verian amenazados su territorios, y vulnerada su tradicional cultura ancestral. Razón por la cual se presentarían conflictos sociales trayendo como consecuencia violencia. No habría paz. En medio de esa situación los programas de educación y capacitación no se podrian desarrollar y la Colectivización de los territorios y el Ordenamiento Territorial para delimitar las fronteras agrícolas y definir el uso de la tierra seguirian en veremos. La cultura campesina continuaría su decadencia (-). (Garay, L. J., Berberi, F., \& Cardona, I; 2010)

\section{Escenario alternativo sobreviviendo.}

En este escenario se corregiría las dificultades sociales y en consecuencia las políticas estatales para el desarrollo del sector agrario estarian pensadas bajo un concepto de desarrollo integral, respetando la autonomía de los pobladores rurales y la visión 
de ellos sobre la construcción de su territorio. Se habrian delimitado las fronteras agrícolas y el uso del suelo mediante un ordenamiento territorial diseñado con los pobladores rurales. (+). Pero habria pocos recursos económicos para invertir en ese desarrollo integral. Lo económico internacional no iría bien. Al haber rechazado y renegociado los TLCS, no acatar la política extractivista de explotación de los recursos naturales ordenada por los organismos rectores del modelo económico internacional, habrian sanciones económicas, por lo que se mermarian las exportaciones. No habría recursos para los subsidios, razón por la cual sería imposible competir con los precios del mercado internacional. Los recursos para la inversión serian muy pocos porque habria un decrecimiento de la economía. La economía tendría serios reveses (-). Es el escenario de la incapacidad. Se tendrian circunstancias a favor, pero no serian suficientes para generar bienestar.

\section{Escenario catastrófico pobreza absoluta.}

Es el escenario de la catástrofe. Se profundizaría negativamente la situación social y politica (-). Por lo tanto no habria politicas estatales para el desarrollo del sector agrario, los campesinos, indigenas, negros, los gremios del sector, y hasta la misma SAC, podrian generar confrontación por los conceptos de territorio y desarrollo, generando escalonamiento del Conflicto Social. El proceso de paz habría fracasado y los excombatientes probablemente retomarian las armas nuevamente agravando la situación de violencia en el país. No habría posibilidad para la Capacitación, la Colectivización de los territorios y el Ordenamiento Territorial acorde con la visión alternativa al modelo avasallador. La cultura campesina continuaría su decadencia. En cuanto a lo económico internacional se presentarian restricciones económicas, los TLC terminarían arruinando mas a los campesinos, indígenas, negros y gremios del sector, haciendo más oscuro el panorama. Cero Subsidios e Inversión para el sector agrario. Estaría amenazada la seguridad alimentaria por el mal uso de la tierra. La explotación indiscriminada de los recursos naturales por la manera irresponsable como se implementa la economía extractiva, causaría daños irreparables e irreversibles al medio ambiente. Seria el escenario de la violencia, el desempleo, el aislamiento. La pobreza absoluta.

\section{Escenario apuesta paz y Prosperidad.}

Sería el escenario ideal. Analizando lo socio político, viviríamos en un país que ha superado el conflicto armado insurgente donde los excombatientes estarian vinculados al desarrollo integral del sector agrario y a las demás instituciones del estado y sectores de la economía. Las politicas estatales para el desarrollo del sector agrario y su componente de concepto de modelos de desarrollo y de territorio, serian diseñadas entre campesinos, Ministerio de Agricultura, el INCODER, los gremios del sector, las SAC y demás actores vinculados, respetando la cultura ancestral de los pobladores rurales como los indígenas, negros y campesinos. Habría pocos Conflictos Sociales entre gobierno y ciudadanos. La Capacitación impartida por las instituciones del estado y las secretarias de la cultura, sería el puntal de reforzamiento de la cultura campesina. La Colectivización de los territorios y el Ordenamiento Territorial serian hechos reales apoyados por las instituciones del estado. (+). Por los lados de lo Económico Internacional: cero corrupciones en el ministerio de agricultura, el banco agrario, el INCODER, gremios del sector y las SAC; en el manejo de los subsidios. Estos llegarian a los campesinos, indígenas y negros. Se materializaría la Inversión de los inversionistas del sector privado y de 
las instituciones del gobierno y del estado en los campos colombianos, con una visión de desarrollo integral. Los Macro proyectos de monocultivos se desarrollarian sin amenazar la seguridad alimentaria y buscando causar los menores daños posibles a los suelos. Tendriamos renegociación de los TLCS haciendo respetar nuestra soberanía y con opciones reales de competitividad. La economía extractiva seria racional sin que los daños causados por sus efectos, sean superiores al beneficio recibido, haciendo uso racional de los recursos naturales a través del buen uso de los suelos con prioridad en el respeto por el Medio ambiente (+) (Insuasty Rodriguez \& Vallejo Duque, 2012)

Matriz DOFA del escenario apuesta.

Analiticamente se han construido las debilidades internas, las oportunidades externas, fortalezas internas y amenazas externas de nuestro escenario apuesta. 
Gráfica 7: Matriz DOFA.

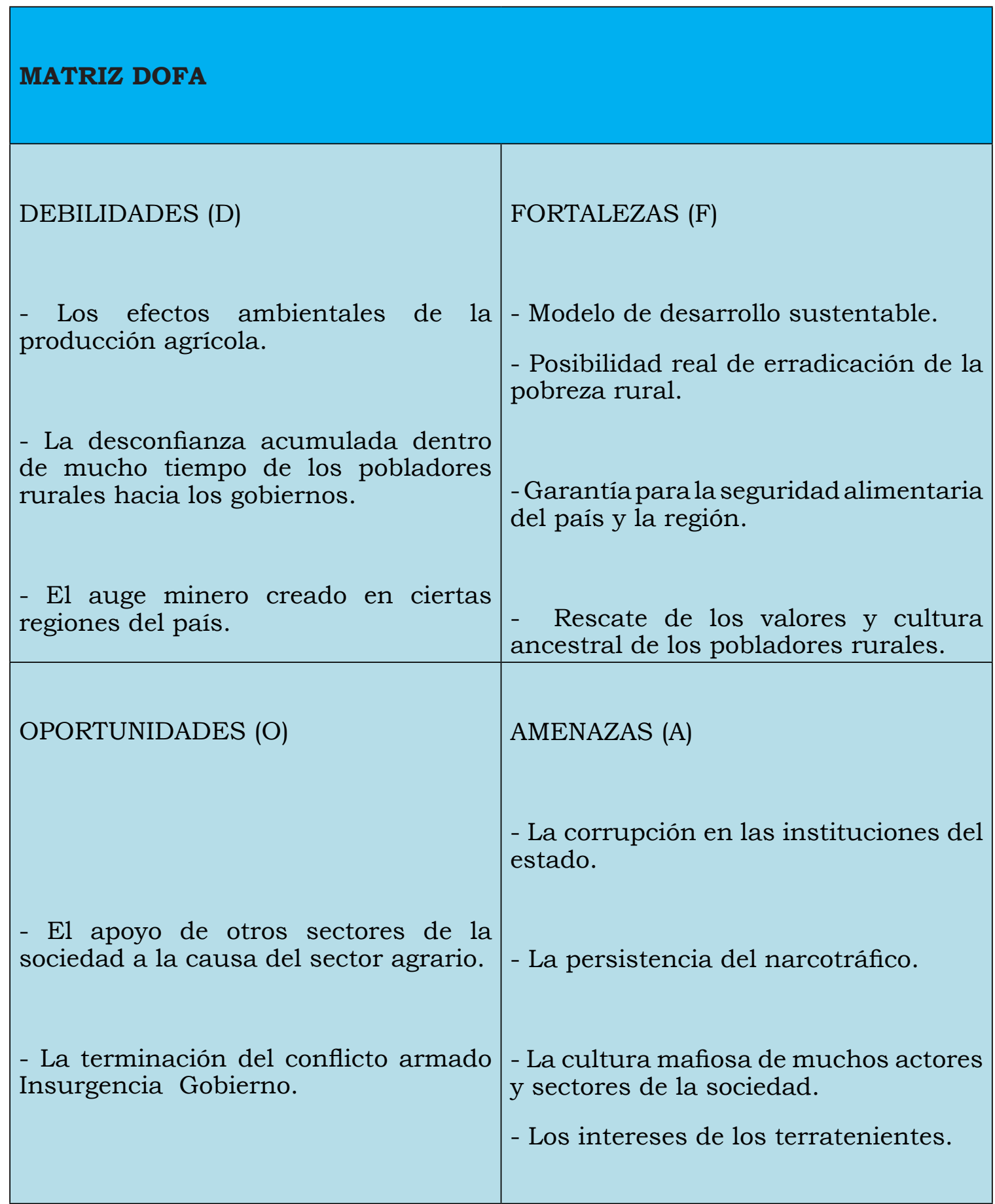

Fuente: elaboración propia 
Gráfica 8: Análisis Matriz DOFA.

\begin{tabular}{|c|c|}
\hline \multicolumn{2}{|l|}{ ANÁLISIS DE LA MATRIZ DOFA } \\
\hline $\begin{array}{l}\text { ESTRATEGIAS DO } \\
\text { (DE SUPERVIVENCIA) }\end{array}$ & $\begin{array}{l}\text { ESTRATEAGIAS FO } \\
\text { (DE CRECIMIENTO) }\end{array}$ \\
\hline $\begin{array}{l}\text { - Buscar comprometer a toda la } \\
\text { sociedad, para que den confianza a los } \\
\text { pobladores rurales de la existencia de } \\
\text { posibilidades reales de resolución a } \\
\text { los problemas del sector. } \\
\text { - Apoyar y acompañar el proceso de } \\
\text { paz. } \\
\text { - Crear conciencia en los pobladores } \\
\text { rurales de las consecuencias de una } \\
\text { economia extractiva basada en la } \\
\text { minería. } \\
\text { - Promocionar el respeto del medio } \\
\text { ambiente y la conservación de los } \\
\text { ecosistemas. }\end{array}$ & $\begin{array}{l}\text { - Aprovechar al máximo las conquistas } \\
\text { del proceso de paz por intermedio } \\
\text { de los instrumentos creados como } \\
\text { resultado de los acuerdos, para exigir } \\
\text { su cumplimiento. }\end{array}$ \\
\hline ESTRATEGIAS (DA) & ESTRATEGIAS (FA) \\
\hline (DE FUGA) & (DE SUPERVIVENCIA) \\
\hline $\begin{array}{l}\text { - Replantear el modelo de implantación } \\
\text { de economías extractivistas. } \\
\text { - Desarrollar acciones con pedagogía } \\
\text { intensiva sobre el tema de la } \\
\text { conservación del medio ambiente. }\end{array}$ & $\begin{array}{l}\text { - Impulsar modelos de desarrollo } \\
\text { sustentable e integral. }\end{array}$ \\
\hline $\begin{array}{l}\text { - Impulsar campañas por el rescate } \\
\text { de los valores éticos y morales. }\end{array}$ & $\begin{array}{l}\text { - Desarrollar programas de capacitación } \\
\text { y educación para los pobladores } \\
\text { rurales. }\end{array}$ \\
\hline
\end{tabular}

Fuente: Elaboración propia

\section{Conclusiones.}

Una importante discusión estaría relacionada con el concepto de los modelos de desarrollo y la visión sobre la construcción de territorios. En tal sentido el modelo de desarrollo no pude ser medido únicamente por el crecimiento en cifras sobre la economía, sino en un modelo de desarrollo sustentable integral que combine el 
bienestar social, el medio ambiente y aspecto económico. En donde no se degrade el planeta y no se comprometa la vida de las generaciones futuras.

En cuanto a concepto de territorio está el otro importante elemento de discusión; el territorio no puede ser comprendido solo como un espacio de gobernanza delimitado geográficamente para la explotación. Si pensamos en un modelo de desarrollo sustentable integral para el buen vivir, el territorio contempla un conjunto de elementos que lo hacen mucho más que eso, es decir como base de las relaciones sociales, en donde hay una permanente construcción social, en donde existe un entramado histórico, cultural y ancestral que lo representa y lo sustenta y que es la base de su visión de futuro.

De otro lado llama la atención que mientras se habla en el mundo de escases de alimentos, se impulsa por parte de los organismos multilaterales, la producción de biocombustibles, poniendo en riesgo la seguridad alimentaria de la humanidad y causando daños irreparables a los suelos en el mediano y corto plazo.

El conflicto armado es la justificación para la no inversión en el campo y esto afecta notablemente su desarrollo, más sin embargo es importante precisar que hay muchos otros elementos que hacen parte de su atraso y que no dependen del conflicto, y que tienen que ver con los modelos de desarrollo que el gobierno colombiano quiere implementar. Se evidencia que el gobierno no está pensando en plantear un modelo de desarrollo para resolver los problemas de los pobladores rurales, sino el de facilitar y permitir la explotación de los recursos naturales por parte de multinacionales mineras, que vienen al país con el único propósito de llevarse los recursos naturales de los colombianos, dejándonos solo los daños ecológicos causados a nuestros territorios y a los ecosistemas.

El gobierno actual ha mostrado intenciones de buscar salidas a algunos problemas puntuales de los campesinos, pero estas no son suficientes. La llamada ley de víctimas y el programa de restitución de tierras, está lejos de ser una alternativa real. A casi cuatro años de estar en aplicación no se han visto los grandes resultados, solo se ven pañitos de agua tibia y los campesinos se quejan de que el problema no es solo de obtener tierras sino de cómo las hacen productivas. En últimas terminan vendiendo sus tierras nuevamente a los terratenientes y el problema de la concentración de la tierra se hace cada vez mayor; generando un problema adicional, el desplazamiento de los trabajadores del campo a las ciudades en busca de otras alternativas de subsistencia.

Hemos logrado identificar los principales problemas que afectan al sector agrario en Colombia relacionados con la concentración de la propiedad de la tierra y su mal uso, falta de vías de penetración hacia las zonas apartadas del campo, rezago tecnológico, altas tasas de interés para préstamos a campesinos, elevados precios de los agroinsumos y fertilizantes, mafias de tras de la comercialización de los productos agrícolas, los tratados de integración y de libre comercio, y en general la falta de una politica gubernamental eficiente que atienda y resuelva las problemáticas del sector.

El uso de la tierra está identificado como uno de los principales problemas. Los territorios campesinos se están utilizando en su mayoria para actividades distintas a la producción de alimentos, minería y macro proyectos de monocultivos de palma de aceite y otros, cuyo 
objetivo es producir biocombustibles; generando con estas prácticas, graves problemas al suelo, al medio ambiente y amenazando la seguridad alimentaria.

Hasta la presente lo expresado por los gremios del sector, los propios campesinos y algunos expertos en temas agrarios, son coincidentes en afirmar que los TLC, y tratados de integración como el llamado Alianza Pacifico, traerán un efecto negativo en el sector, en el entendido que nuestros campesinos no están en la capacidad de competir con los productores de alimentos de países industrializados, en donde estos pueden producir a precios más bajos, y con el apoyo de subsidios por parte de los gobiernos como es el caso de los estados unidos.

Podemos decir que las variables claves para el cumplimiento del escenario apuesta está en el rediseño de las políticas estatales para el desarrollo del sector, políticas estas que tendrán que ser pensadas en un modelo de desarrollo integral de los territorios campesinos. En tal sentido es importante recoger las propuestas elaboradas por los propios pobladores rurales realizadas en sus cumbres agrarias, así serian proyectos elaborados con la participación de los pobladores rurales, conocedores de su propia realidad y actores importantes y estratégicos en la elaboración de cualquier plan.

De ahí se desprenden una serie de variables como la destinación de recursos para la inversión en el sector, el apoyo económico a través de subsidios para los productores, la capacitación entendida como la formación intelectual y técnica para los pobladores rurales, la manera como se implementen los macro proyectos de monocultivos, la construcción de una verdadera paz, la cultura campesina, el uso de los recursos naturales, el conflicto social existente entre la sociedad, gobierno y empresas multinacionales, el concepto de territorio, la colectivización de los territorios o la propiedad colectiva de territorios, el medio ambiente ligado a la conservación de los ecosistemas, el uso de la tierra y de los territorios, la economía extractiva relacionado esto con la explotación indiscriminada de los recursos naturales y la concesión de tierras que tiene que ver con el tema de la restitución de tierras.

Después de haberse intentado tantas veces en Colombia un programa efectivo de reforma agraria, seguimos esperando que este algún día sea posible. Está claro que esto sería un paso importante para iniciar el camino de la búsqueda de soluciones reales, pero no sería solo entrégales tierras a los campesinos, esto de nada serviría si el campesino no tiene dinero para invertir y no está garantizada la comercialización de su producción.

Como hemos visto en las tendencias mundiales en cuanto a las experiencias obtenidas en países donde se terminó el conflicto y se plantearon salidas a las crisis del sector agrario, estas nos muestran que los programas trazados no se materializaron, pues los gobiernos incumplieron los acuerdos y los programas que serian para los pobladores rurales, fueron aprovechados por los terratenientes y los industriales y grandes empresarios de la producción de alimentos. Generando incluso mayor pobreza y explotación a los pobladores del campo.

Una posible alternativa a esta situación está en lograr que los pobladores rurales se agrupen en territorios colectivos, y hagan una defensa colectiva de los mismos, esto marcaria una diferencia con lo visto en El Salvador y Guatemala. De ahí la 
importancia de la implementación de las zonas de reserva campesina o figuras similares que sería un instrumento desde donde los pobladores rurales podrian organizarse, exigir el cumplimiento de los acuerdos pactados, y construir desde su propia visión respaldados en su autonomía, verdaderos modelos de desarrollo alternativos bajo nuevos conceptos de territorio. 


\section{Referencias Bibliográficas.}

Acción Social. (2009). Agencia Presidencial para la Acción Social y la Cooperación Internacional (Acción Social) Proyecto de protección de tierras y patrimonio de la población desplazada (PPTP). Caracterización de las reservas forestales de la Ley 2 de 1959. Bogotá: Serie Consultorias e Investigaciones.

Aeidl. (1997). Innovación y desarrollo rural. Comisión Europea, Observatorio Europeo de Desarrollo Rural. Bruselas.

Adepro. (3 de 1 de 2001). Segunda parte: El Salvador al final del Siglo XX. Obtenido de Adepro, especialistas en Desarrollo local: http://www.adepro.biz/espanol. php?id=6\&book $=5$

Alasdair , C. (28 de 2 de 2005). El Rol de las Organizaciones Comunitarias en la. Obtenido de adipsa: http://adipsa.org/downloads/ADIPSA_Estudio_de_Caso.pdf

Aráoz, C. (2002). Curso Desarrollo Rural (DRU) 2002 FODEPAL. RLC-FAO. Santiago de Chile.

Banco Mundial. (28 de 02 de 2013). Tasa de incidencia de la pobreza, sobre la base de la línea de pobreza rural (\% de la población rural). Obtenido de Banco Mundial: http://datos.bancomundial.org/indicador/SI.POV.RUHC/countries

Bandira Greño, P., Atance Muñiz, I., \& Sumpsi Viñas, J. (2004). Las políticas de desarrollo rural en América Latina. Requerimientos de un nuevo enfoque. Cuadernos de Desarrollo Rural.(51), 115-136. Obtenido de http://www. javeriana.edu.co/ier/recursos_user/documentos/revista51/115_136.pdf

Bardhan, P. (2001). Institucionalidad, reformas y desempeño de la agricultura. En Kostas Stamoulis (ed.) Alimentación, agricultura y desarrollo agrícola. FAO. Roma.

Ballara, Marcela; Parada, Soledad 2009. El empleo de las mujeres rurales. Lo que dicen las cifras.Santiago: Cepal-fao.

De Ferranti, David; Perry, Guillermo; Foster, William; Lederman, Daniel; Valdés, Alberto. (2005). Beyond the city: the rural contribution to development. Washington DC: Banco Mundial.

Departamento Nacional de Planeación (DNP). (2010). Politica nacional para la interrelación del catastro y el registro. Documento Conpes 3641. Bogotá: febrero 15.

Documento sobre la situación del Agro desarrollo rural desde el acuerdo de Paz en Guatemala. (28 de 02 de 2000). Obtenido de landcoalition.org: http:// www.landcoalition.org/sites / default/files/legacy/legacypdf/ARrO0congcoop. pdf?q=pdf/ARrO0congcoop.pdf 
Garay, L. J., Berberi , F., \& Cardona, I. (2010). Impactos del TLC con Estados Unidos sobre la Economía Campesina en Colombia. Bogotá: Ilsa. Obtenido de http://ilsa.org.co:81/biblioteca/dwnlds/otras/tlc/cont.pdf

Godet, Michel. (1993). "De la anticipación a la acción. Manual de prospectiva estratégica", Marcombo Editores, Barcelona.

Goebertus, Juanita. (2008). Palma de aceite y desplazamiento forzado en Zona Bananera: 'trayectorias' entre recursos naturales y conflicto. Colombia Internacional (67).

INCODER. (05 de 05 de 2013). Instituto colombiano para el Desarrollo Rural. Obtenido de incoder.gov.co: http://www.incoder.gov.co/portal/default.aspx

Insuasty Rodriguez, A., \& Vallejo Duque, Y. (2012). Crar Ambiente para la Paz. Kavilando, 11-18.

Lafaurie Rivera, José Félix. (2010). La tierra y el desarrollo rural en Colombia. Una propuesta de política pública. Bogotá: Fedegán.

Mejia, Daniel; Restrepo, Pascual. (2008). The war on illegal drug production and trafficking: an economic evaluation of Plan Colombia Documento cede (19). Bogotá: Universidad de los Andes.

Mesa de Conversaciones Habana 2014. (21 de 07 de 2013). Primer informe conjunto de la Mesa de Negociaciones entre el Gobierno de Colombia y las Fuerzas Armadas Revolucionarias de Colombia Ejercito del Pueblo, 2013. Obtenido de mesadeconversaciones.com.co:

https://www.mesadeconversaciones.com.co/sites/default/files/Primer\%20 informe $\% 20$ conjunto $\% 20 \mathrm{de} \% 201 \mathrm{a} \% 20 \mathrm{Mesa} \% 20 \mathrm{de} \% 20$ Conversaciones $\% 20$ entre\%20 e1\%20 Gobiernode\%201a\%20Rep\%C3\%BAblica\% 20 de \% 20 Colombia\%20y\%20las\%20Fuerzas\%20Armadas\%20Revolucionarias\%20de\%20 Colombia $\%$

Pnud. (01 de 01 de 2011). Colombia Rural. Razones para la esperanza. Obtenido de planipolis.iiep.unesco.org: http:// planipolis.iiep.unesco.org/upload/Colombia/ Colombia_NHDR_2011_resumen.pdf

Spyros Makridakis y Steven C.. Wheelwright. (1989). Manual de Técnicas de Pronósticos (The Handbook of Forecasting). Ed. Noriega y Limusa. México.

Schwartz, P. (21 de 11 de 1995). La planificacion estrategica por escenarios. Obtenido de cuadernosadm.univalle.edu.co:

http: / / cuadernosadm.univalle.edu.co/Articulos / 21 -Cuadernos_de_ Administracion_(Noviembre-1995)/21-(12)_La_planificacion_estrategica_por_ escenarios_(Peter_Schwartz).pdf 
Uprimny, Rodrigo; Chaparro, Sergio; Guzmán, Diana Esther; Sánchez, Camilo. (2010). Mujeres, sector rural y tierras en Colombia. Bogotá: Unifem, Pnud, DeJusticia,

Wladimir M. Sachs. (1980). Diseño de un Futuro para el Futuro. Un ensayo sobre los métodos e importancia de la planeación prospectiva. Ed. Fundación Javier Barros Sierra, A.C. $2^{a}$ edición corregida. México. 